\title{
Feedback of real-time fMRI signals: From concepts and principles to therapeutic interventions
}

Moses O. Sokunbi ${ }^{1^{*}}$

${ }^{1}$ Cognitive Neuroscience Sector, International School for Advanced Studies (SISSA),

Via Bonomea 265, 34136 Trieste, ITALY.

*Corresponding author:

Moses O. Sokunbi

Cognitive Neuroscience Sector,

International School for Advanced Studies (SISSA),

Via Bonomea 265, 34136 Trieste,

ITALY

Tel: +39040 3787602

Email: $\underline{\text { msokunbi@sissa.it }}$ 


\begin{abstract}
The feedback of real-time functional magnetic resonance imaging (rtfMRI) signals, dubbed "neurofeedback", has found applications in the treatment of clinical disorders and enhancement of brain performance. However, knowledge of the basic underlying mechanism on which neurofeedback is based is rather limited. This article introduces the concepts, principles and characteristics of feedback control systems and its applications to electroencephalography (EEG) and rtfMRI signals. Insight into the underlying mechanisms of feedback systems may lead to the development of novel feedback protocols and subsystems for rtfMRI and enhance therapeutic solutions for clinical interventions.
\end{abstract}

Keywords: Brain-computer interface, clinical disorders, feedback system, neurofeedback, real-time fMRI, self-regulation.

\title{
I. Introduction
}

Real-time functional magnetic resonance imaging (rtfMRI) enables the immediate visualization of brain activations as they are being acquired. This is possible because of the availability of higher-field MRI scanners, fast data acquisition sequences, improved and immediate pre-processing of functional magnetic resonance images (fMRI), improved statistical analysis techniques and improved methods of visualization of brain activations [1]. These developments made the feedback of rtfMRI signals possible, thereby allowing for fMRI brain-computer interfacing (fMRI-BCI) [1] popularly known as neurofeedback [2, 3]. RtfMRI can be applied as a clinical neuroimaging tool for diagnosis, monitoring of disease, tracking of therapeutic response and as a therapeutic tool via the feedback of rtfMRI signals $[4]$. 
Neurofeedback is a training method whereby a person receives continuously real-time information about changes in neural activity in certain brain region, which they use to learn self-regulation and control of the neural activity in that target region so as to produce changes in behaviour [4]. Various techniques have been used to present visual feedback for rtfMRI neurofeedback. For example, scrolling time series graphs [5-7], the thermometer bars and a 3D animated character (a fish in water) [8], functional maps of the brain [9], video-based feedback [10] and the changing size of food pictures [11] have been used.

The design of the feedback system is therefore an important factor to consider for an efficient fMRI-BCI/neurofeedback system since temporal delay of feedback information is relevant for learning and training participants to self-regulate their blood oxygen level dependent (BOLD) fMRI signals. The feedback component is the core of an efficient fMRIBCI/neurofeedback system. However, information about the underlying concepts, principles and characteristics governing the fMRI-BCI/neurofeedback process in the fMRI neurofeedback literature is rather limited. This article takes a look at the nature and characteristics of feedback systems and the application of this approach to rtfMRI neurofeedback systems. It is hoped that insight into the basic concepts, principles and characteristics of feedback systems will lead to improved design and implementation, and to the development of new tools for rtfMRI neurofeedback as a therapeutic tool and enhancement of brain performance.

\section{What is a feedback system?}

Consider a process represented by a rectangular block with an input and output signal as shown in Fig. 1A. To control (perform an operation on) the process, another rectangular block (called the controller) is added before the process in Fig. 1B, and its output (control 
signal) is used as input to the process, to obtain the desired response. The controller is responsible for characterizing the functional relationship among the components of the system. Fig. 1B is an open-loop control system [12]. When the output from the process in Fig. 1B is connected to a small circle called a summing point entailing a reference input signal as shown in Fig. 2, a feedback path is developed. The feedback path enables the output signal from the process to be compared with the reference input signal to the system so as to obtain the appropriate control action which is a function of the output and input signals. Fig. 2 is a closed-loop feedback control system [12]. The following are the components of the feedback control system depicted in Fig. 2 [12]:

- The process is the subsystem controlled by the feedback control system

- The controlled output signal is the output of the process due to the control exerted by the feedback control system

- The feedback element such as sensors/transducers of the controlled output relates the controlled output to the feedback signal $\boldsymbol{b}$.

- The feedback path is the transmission path from the controlled output to the summing point.

- The feedback signal $\boldsymbol{b}$ is a function of the controlled output.

- The reference input signal $\boldsymbol{r}$ is an external signal or stimulus applied to the summing point of the feedback control system in order to exert control on the process.

- The error signal $\boldsymbol{e}$ is the result of the addition or subtraction of the reference input signal and feedback signal. The error signal generates the control action of the feedback control system and it serves as input to the controller.

- The control signal is the output signal of the controller and it serves as input to the process. 
- The forward path is the transmission path from the summing point to the controlled output.

- When the summing point is an adder $(e=r+b)$ the system is a positive feedback control system.

- When the summing point is a subtractor $(e=r-b)$ the system is a negative feedback control system.

In the context of a biological system, such as a brain computer interfacing (BCI) system, Fig. 1B represents an open-loop BCI system while Fig. 2 is a closed-loop BCI system.

\section{Characteristics of feedback systems}

Systems with a feedback path normally exhibit some of the following properties:

- Feedback systems have the ability to exert control on the systems [12].

- Regulatory ability: A feedback system is able to embark on self-regulation to obtain a desired behaviour by ensuring that the measured output is equal to or approximately equal to the reference input [13].

- Adaptability: The system can adapt to changes due to new experiences

- Increased accuracy: The system can increase accuracy by ensuring that the measured output converges to the reference input in the case of regulatory control, disturbance rejection and optimization objective [13].

- Stability: Feedback can be used to change the dynamics of a system. The behaviour of a system can be altered through feedback to meet the needs of an application [14]. This implies that unstable systems can be stabilized, sluggish systems can be made responsive and systems with drifting operating points can be held constant. 
Improperly designed [14] or impaired systems can tend towards oscillation or instability [12].

- With feedback there are reduced effects of nonlinearities [12].

- Increased bandwidth: Feedback helps to increase the frequency response of a system to variations in the input signal [12].

- Robustness: A feedback system is robust to uncertain external perturbations because it is able to implement a corrective action from the error signal obtained from the difference between the sensed regulated signal and its desired signal level. This enables the system to return to the desired operating point [14].

- Learning, higher levels of abstract reasoning and automation: Feedback can enhance learning, abstract reasoning and higher levels of automation. These are possibilities in the domain of artificial intelligence where there is a greater role for dynamics, robustness and interconnections in numerous applications [14].

- Optimization: A system with feedback can optimise its performance by obtaining the "best" value of the measured output [13].

\section{Feedback of Biological or Physiological systems}

Biological or Physiological systems such as the human body entail the integration of feedback loops, complex networks of control systems and other regulatory mechanisms that enable it to function normally [15]. The control systems of the human body exist at many levels of organisation. The intercommunication among the electrical, chemical and mechanical components of these systems enable a constant information exchange among the control systems necessary for normal and adaptive functioning [15]. The degradation of any of the components of these control systems may lead to the loss of adaptive capacity of the system that can cause disease-related functional impairments [15]. Some examples of these 
physiological control systems are blood pressure, heart rate, brain electrical activity and BOLD signal, hormone concentrations, balance and gait [15].

A simple example of a biological control system involved in walking in a prescribed direction [12] is depicted in Fig. 3. Here, the input signal represents the desired walk direction while the output is the actual walk direction. The feedback signal is a function of the actual walk direction. The summing point detects the error signal which is the difference between the desired and actual walk directions. This difference is sent to the brain, the controller of the walking system, which controls, for example, the legs and feet to walk in the prescribed direction. From this point of view, walking is a closed-loop operation because the control action is a result of the difference between the desired and actual walk directions. The feedback loop is broken if the eyes are closed and the system becomes open-loop.

Human brain activity can be measured non-invasively in a number of ways such as magnetoencephalography (MEG), electroencephalography (EEG), near infrared spectroscopy (NIRS) and BOLD fMRI, just to mention a few. However, of these only EEG and rtfMRI have been commonly used for neurofeedback experiments and training. Therefore, in the following sections we shall discuss briefly EEG based, rtfMRI based and simultaneous rtfMRI-EEG based neurofeedback techniques.

\section{Feedback of EEG signals}

The earliest neurofeedback studies were implemented using electroencephalography (EEG), which entailed the feedback of electrical brain activity [16]. EEG neurofeedback, also popularly known as biofeedback [17], entails the feedback of extracted EEG electrophysiological signals in a closed-loop fashion. The EEG electrophysiological signals 
are extracted when subjects are exposed to stimuli or execute mental tasks while their cortical activity is being recorded by EEG [18]. These electrophysiological signals are regulated by the subjects during EEG neurofeedback. The electrophysiological signals that may be used for EEG neurofeedback are the Slow Cortical Potential, Sensorimotor Rhythms, Visual Event-Related Potentials (e.g. the P300) and Steady-State Visual Evoked Potentials [18]. EEG biofeedback has been used in the treatment of attention deficit hyperactivity disorder (ADHD) [17]. Many randomised controlled trials have been reported in the treatment of ADHD using EEG based neurofeedback [19]. Other treatment areas are in epilepsy [20] and mood disorders such as anxiety disorder [21]. Other indications of the application of EEG biofeedback are to conditions such as fibromyalgia, migraine, chemical dependency and syndromes related to traumatic brain injury [17]. EEG biofeedback has also been used in healthy individuals to improve performance such as in artistic aspects of music [22]. The biggest advantages of EEG are that it has good temporal resolution (in the millisecond range), and that it is inexpensive and portable in comparison to fMRI. However, poor spatial resolution and the inverse problem issue are some of the properties that limit the clinical applications of EEG neurofeedback. The EEG inverse problem occurs when some currents produce potentials that cancel out each other thereby making it impossible to reconstruct a unique solution for a given EEG signal. Notwithstanding the limitations of fMRI such as poor temporal resolution and the slow hemodynamic response, rtfMRI can be used to map the whole brain thereby providing the possibility to target specific brain regions and networks with improved anatomic precision compared to EEG that is mostly limited to cortical regions.

\section{Feedback of fMRI signals}

The temporal delay associated with the feedback of BOLD fMRI signals is critical to the success of rtfMRI neurofeedback experiments because it can affect the ability of the 
participant to self-regulate the signals [23]. In most rtfMRI neurofeedback studies, feedback has been continuously presented to the participant with minimum delay, about $2 \mathrm{~s}$ [23]. Other studies have averaged the feedback signal over a longer period to achieve meaningful information, up to 1 minute in length [9]. Operant conditioning (contingency and contiguity) is beneficial to the delivery and self-regulation of the feedback signal. Temporal contiguity implies the time interval between response and reinforcement [23]. The intermittent (at the end of the regulation phase) presentation of feedback (about $20 \mathrm{~s}$ delay) is more effective than continuous (at each volume acquisition rate) presentation (about 2 s) when an imagerybased strategy is used for self-regulation [24]. In certain situations such as the early stage of learning, the intermittent presentation of the feedback signal could be more advantageous as it does not interfere with the ongoing imagery during self-regulation [24]. Contingency is the conditional probability of reinforcement due to a response or failure to respond. The contingency of the rtfMRI feedback signal is often manipulated as a control condition i.e. a sham feedback [25]. Other factors like distraction from the thermometer used for presentation of the feedback signal can decrease successful learning, especially during dual-task effects associated with monitoring the feedback signal during cue exposure [26]. A typical architectural setup for rtfMRI neurofeedback used for therapeutic purposes is described in the following section.

\subsection{Real-time fMRI neurofeedback architecture}

The real-time fMRI neurofeedback architecture and operation consists of two set-up runs; the localiser and neurofeedback runs. The localiser run entails the signal acquisition and signal analysis subsystems while the neurofeedback run includes the signal feedback subsystem in addition to the signal acquisition and analysis subsystems. 


\subsubsection{Localiser run:}

The localiser run is the first step for carrying out neurofeedback training. It is used to discriminate the region of interest (ROI) that would be used for subsequent neurofeedback runs. Selecting a ROI typically depends on the expected behavioural output which can be achieved by using previous knowledge of neural mechanisms underlying the expected behavioural effect [23]. For example, to control the perception of pain a rtfMRI neurofeedback experiment regulated BOLD activations in the rostral anterior cingulate cortex (rACC) [25]. Specific ROIs can be either functionally or anatomically defined [4]. A ROI may be functionally defined through the GLM based method which uses the average BOLD response in that ROI [23]. The GLM based method allows for nuisance parameters to be regressed out and the average BOLD signal at each voxel in the ROI to be obtained from the residual of the GLM [23]. It may also be defined by using the differential activity in two ROIs [27]. There are also ongoing studies which are exploring the possibility for feedback of functional and effectivity connectivity between brain areas [23]. Some ROIs can be anatomically defined based on brain atlases or macroscopic anatomical landmarks, for example the insular cortex [28]. ROIs are usually difficult to define anatomically due to high variability, however, anatomical localisers may be more appropriate in situations where it is difficult to define a ROI functionally, like the substantia nigra and where the ROI is well defined anatomically [23]. Also, a combination of overlapping anatomical and functional localisers may help improve the definition of ROIs [23]. Some evidences have shown that functional localisers produce a better contrast-to-noise signal in some situations when compared to anatomical localisers but provided there is minimal head movement [23].

A ROI may also be functionally defined using multivariate pattern analysis (MVPA) methods [29]. MVPA methods use supervised learning techniques such as support vector machines to 
determine the optimal set of weights from a delineated ROI or the whole brain to combine the BOLD signal of voxels into a single neurofeedback score [4]. ROIs can be adaptively defined especially when it involves training over time such as in different neurofeedback runs and sessions among different days [30]. This will enable the system to allow for potential brain changes related to training over time, which could arise as a result of individual differences in optimal learning strategies and performance [4]. These brain changes may result in changes in the level of activation in the defined ROIs and recruitment of different neural networks to enhance improved performance [4]. The optimal ROI size may be obtained by dragging a rectangle around "significant" pixels in a multi-slice or single slice view of the brain during or after real-time processing [31]. The averaged time course of the significant voxels within the ROI represents the fMRI signal strength with respect to time [31]. Here, we will briefly describe the GLM method for defining ROIs.

The first step in the GLM based method for localising ROIs to be used for subsequent neurofeedback runs is the signal acquisition subsystem. In the localiser run, brain activity of a participant to a specific set of stimuli or to a specific task is acquired using an MRI scanner. For example, in Linden et al. [30], an ROI was identified by the contrast between responses to positive and neutral images when patients with depression passively viewed positive, negative and neutral images obtained from the International Affective Pictures System (IAPS) [32]. Linden et al. [30] assessed brain responses to positive, negative and neutral pictures by presenting four pictures of the same emotion category in blocks of 6 s i.e. $1.5 \mathrm{~s}$ per picture, alternating with a fixation baseline of $12 \mathrm{s.} 12$ blocks per category were presented in pseudorandom order. The pictures used showed scenes of danger or disgust in the negative category and scenes of romance including mild erotica or exciting sports in the positive category. They identified a target area by the contrast between responses to positive and 
neutral images in the localiser run to ensure that an area involved in positive emotion processing was selected [30]. Also, Sokunbi et al [11] determined an ROI related to processing of food-related visual stimuli, by comparing activity to the target stimuli (food pictures) and neutral control stimuli (household objects) while healthy participants viewed images on the MRI projector screen. Image reconstruction and distortion correction to improve signal to noise ratio were performed by the MRI scanner computer. The fMRI images after signal acquisition pre-processing were stored in the MRI image pool.

The second step in the GLM method for localising ROIs is the signal analysis subsystem, which can be implemented with Turbo-Brainvoyager (TBV) (Brain Innovation, Maastricht, The Netherlands; [31]). The reconstructed fMRI images are retrieved from the MRI image pool by TBV, which performs real-time 3D motion correction, temporal filtering, spatial normalisation, spatial smoothing and real-time statistical analysis via an incremental GLM. A static ROI is selected by drawing an area of the respective contrast of interest on the functional map (3D BOLD signal) computed by the TBV. The potential areas to be selected as a ROI, for example in Linden et al.[30] and in Sokunbi et al [11] are limited to areas responsive to positive emotions such as the insula and the ventrolateral prefrontal cortex [30], and to motivational brain areas such as amygdala and insula [11] respectively. Voxels are included in the target ROI for signal extraction at an investigator chosen t-statistical threshold of between 2 and 3. TBV extracts average BOLD signal values (betas) from the ROI and stores them in continuously updated real-time protocol files (rtp files). The storage of these rtp files concludes the localiser run used for discriminating the target ROI and it lasts for about 10 minutes, which is the time it takes for the images to be presented to the participant. The localiser run is an open-loop system which does not include a signal feedback subsystem. 
Fig. 4 depicts a schematic representation of the localiser run for an fMRI neurofeedback architecture.

\subsubsection{Neurofeedback run:}

A neurofeedback run entails a signal feedback subsystem in addition to a signal acquisition and signal analysis subsystems, and it is a closed-loop system. The signal feedback stage involves retrieving the stored rtp files for the ROI selected during the localiser run and mapping the percentage change in BOLD signal values of the ROI on a feedback presentation tool such as the thermometer bar [30], or food pictures of changing size [11] across three consecutive TRs. PsychoPy [33] is one of the frequently used softwares used for the signal feedback analysis and presentation. In Linden et al [30], a 1\% increase of the relative BOLD signal was set as maximum level for the thermometer, with each of the 10 red thermometer bars corresponding to $0.1 \%$ BOLD signal change. Participants are informed about the hemodynamic delay, i.e. that it would take between 4-8 seconds for any changes in brain activity to lead to a change of the BOLD signal and thus of the thermometer. Minimising the delay is however critical for volitional control [10]. Feedback is presented to the participants with a delay that depends on the time for signal acquisition, signal analysis and signal feedback processing and presentation.

During a neurofeedback run, the stored ROI is uploaded by the signal analysis software (TBV) at the signal analysis subsystem level. At the signal feedback subsystem level, the stored rtp file for this ROI is retrieved and the percent BOLD signal change of the ROI is mapped onto a feedback presentation tool. For example, using a thermometer with ten bars as the feedback presentation tool similar to Linden et al. [30], during self-regulation training, the participant is asked to upregulate (increase) the red bars of the thermometer to the maximum 
number of bars (10 bars) when he/she sees the thermometer superimposed on a green background. When the green background changes to yellow after 20 seconds, the participant is asked to downregulate (decrease) the red bars to their minimum (no red bars). This alternation of presenting the thermometer on a green background (upregulation) or on a yellow background (downregulation) lasts for 20 second in each case and continues till the end of the neurofeedback run, which is 3 minutes. As the participant is engaged in the up and down regulation tasks, the signal acquisition subsystem is also running simultaneously, acquiring functional images of the self-regulation training, which are analysed at the signal analysis subsystem (TBV), hence forming a close-loop system (Fig. 5). During the upregulation and downregulation tasks participants are encouraged to use strategies that would help them to achieve the aims of the tasks. Fig. 5 shows a schematic representation of a neurofeedback run using thermometer bars as the feedback presentation tool.

Alternative software that is also used for real-time fMRI neurofeedback is the Functional Real-time Interactive Endogenous Neuromodulation and Decoding (FRIEND) toolbox which is freely available software [2]. FRIEND is a graphical user interface (GUI) user friendly toolbox for real-time fMRI processing, multi-voxel pattern analysis and decoding by support vector machines (SVM) and rtfMRI neurofeedback [2].

\subsection{Characteristics of rtfMRI neurofeedback as a clinical neuroimaging tool}

RtfMRI neurofeedback has some characteristics which enable it function as a clinical neuroimaging tool. These characteristics are brought about by the combination of several technical, neuroscientific and clinical issues in the implementation of the localizer and neurofeedback runs. Some of these characteristics are: 
- Learning: rtfMRI neurofeedback can induce learning by providing feedback information. Learning is said to be induced when experience influences behaviour and alters brain structure or function [4]. Experience may consist of elements of the task, feedback about regional brain activation and cognitive processes that arise due to the task [4]. With regards to behaviour, learning may entail memory recall, recognition, improved perception, priming and motor action [4].

- Robustness: It can be used as a testing tool; here it can be used to test the robustness of neurobiological hypotheses before a more invasive procedure can be embarked on [4]. For example, in testing the efficacy of deep brain stimulation which is an invasive procedure for the treatment of depression [34], rtfMRI neurofeedback can be used to test this hypothesis before it is implemented invasively [4].

- Adaptability: RtfMRI neurofeedback training enhances adaptability which leads to improvement on the task used during training and behavioural effects [4]. For example, training self-regulation of activity in the dorsal anterior cingulate cortex (implicated in pain perception and regulation) led to a change in the perception of pain in patients with chronic pain [25].

- Performance: It can be used to enhance performance such as in the validation of sensorimotor rhythm, beta and alpha-theta protocols for improving attention, memory, mood, music and dance performance in healthy participants by using cognitive and neurophysiological measures [35].

- Optimization: It can be used to promote the optimal control of cognitive control strategies. For example, rtfMRI neurofeedback was used to acquire an optimal cognitive control strategy for healthy participants who successfully learned controlling their visual cortex activity [36]. 


\subsection{Therapeutic interventions using rtfMRI neurofeedback systems}

RtfMRI has many potential applications as a clinical neuroimaging tool however the technique has been extensively used to study and alter brain function and behaviour via the feedback of rtfMRI signals [4]. RtfMRI neurofeedback has been piloted for investigation as a therapeutic tool for the treatment of some clinical disorders and syndromes such as chronic pain [25], schizophrenia [37], addiction [38], attention deficit hyperactivity disorder (ADHD) [39], stroke [40], tinnitus [41], obesity [42] and depression [30]. Further studies reported the applications of rtfMRI neurofeedback to other clinical disorders [3, 43-45]. In healthy participants, we have recently applied rtfMRI neurofeedback to influence the emotion regulation network of children and adolescents [46].

The design and development of novel feedback protocols and subsystems may enhance better therapeutic interventions in the application of rtfMRI in a clinical setting. Recently, we developed a novel motivational feedback subsystem for the regulation of visual cue reactivity [11]. The subsystem provides simultaneous feedback through the changing size of food pictures, which are mapped to the magnitude of fMRI signal change from a target brain area related to motivational processes such as craving or hunger [26]. Our novel approach has the advantage that the feedback-guided self-regulation is based on visual changes in the stimulus responsible for the targeted brain responses, thereby minimising distracting/dual effects associated with monitoring the feedback stimulus during cue exposure [11]. This enables the brain to better adapt to the changing size of food pictures presented as task stimulus and feedback signal. Our approach also included mirror runs to control for physical/perceptual confounds such as habituation [11]. This novel motivational feedback subsystem may find therapeutic application in pathologies such as obesity or addition. 
Recently, Fovet et al. [47] proposed a methological approach of using rtfMRI neurofeedback to treat auditory-verbal hallucinations $(\mathrm{AVH})$, a condition prevalent in patients suffering from schizophrenia. Here, they propose three strategies of relieving AVH with rtfMRI neurofeedback. The strategies are using a priori target localised using structural MRI, defining the ROI using a functional localiser using a priori hypothesis and using pattern recognition by a multivariate classifier. The activity patterns concomitant to hallucinations from these strategies can then be fed back to the patients for treatment purposes [47]. They suggested proof-of-concept studies on their approach which may establish a new brain imaging-guided psychotherapy technique for patients that are unresponsive to conventional treatments. Also, Gerin et al. [48] implemented a novel rtfMRI neurofeedback intervention to control amygdala activity in war veterans with chronic post-traumatic stress disorder (PSTD).

\section{Simultaneous rtfMRI-EEG neurofeedback}

A combination of the superior temporal resolution of the EEG and the good spatial resolution of the fMRI can be exploited in a simultaneous rtfMRI-EEG neurofeedback platform with the aim that this hybrid outcome will lead to better neuroadaptive effects and new clinical solutions. This novel approach has been recently implemented for the very first time in a proof-of-concept study by Zotev et al. [49]. These authors demonstrated the feasibility of simultaneous emotional self-regulation of both hemodynamic and electrophysiological activities in healthy participants performing a positive emotion induction task based on retrieval of happy autobiographical memories. Using the rtfMRI-EEG neurofeedback framework, participants successfully and simultaneously self-regulated their BOLD fMRI activation in the left amygdala and frontal EEG power asymmetry in the high-beta band [40]. These findings suggest potential applications of rtfMRI-EEG neurofeedback approach for the 
development of novel cognitive neuroscience research protocols and better treatment interventions in psychiatric conditions such as depression [49].

\section{Conclusions}

This paper considers the basic concepts, principles and characteristics of feedback systems and how this relates to biological or physiological systems such as the human brain. Furthermore, the feedback of EEG signals, fMRI signals and simultaneous EEG - fMRI signals for treatment of clinical disorders are considered. It is expected that potential application of the characteristics of feedback systems may lead to the design and development of novel feedback protocols and subsystems for rtfMRI and enhance therapeutic solutions for clinical interventions.

\section{Conflict of interest}

There is no conflict of interest.

\section{Acknowledgments}

I am grateful to Prof David E.J. Linden of Cardiff University, Cardiff, Wales, United Kingdom from whom I learnt about the practical realities of adaptive feedback control system as applied to the human brain in health and disease. 


\section{References}

[1] Weiskopf N. Real-time fMRI and its application to neurofeedback. Neuroimage 2012; 62: 682-692.

[2] Sato JR, Basilio R, Paiva FF, Garrido GJ, Bramati IE, Bado P, Tovar-Moll F, Zahn R, Moll J. RealTime fMRI Pattern Decoding and Neurofeedback Using FRIEND: An FSL-Integrated BCI Toolbox. PLoS ONE 2013; 8(12): e81658. doi:10.1371/journal.pone.0081658.

[3] Birbaumer N, Ramos Murguialday A, Weber C, Montoya P. Neurofeedback and braincomputer interface clinical applications. Int Rev Neurobiol. 2009; 86:107-17.

[4] Stoeckel LE, Garrison KA, Ghosh S, Wighton P, Hanlon CA, Gilman JM, Greer S, Turk-Browne NB, deBettencourt MT, Scheinost D, Craddock C, Thompson T, Calderon V, Bauer CC, George M, Breiter HC, Whitfield-Gabrieli S, Gabrieli JD, LaConte SM, Hirshberg L, Brewer JA, Hampson M, Van Der Kouwe A, Mackey S, Evins AE. Optimizing real time fMRI neurofeedback for therapeutic discovery and development. Neuroimage Clin. 2014; 5: 24555.

[5] Weiskopf N, Veit R, Erb M, Mathiak K, Grodd W, Goebel R, Birbaumer N. Physiological selfregulation of regional brain activity using real-time functional magnetic resonance imaging (fMRI): methodology and exemplary data. Neuroimage 2003; 19: 577-586.

[6] Weiskopf N, Scharnowski F, Veit R, Goebel R, Birbaumer N, Mathiak K. Self-regulation of local brain activity using real-time functional magnetic resonance imaging (fMRI). J Physiol Paris 2004; 98: 357-373.

[7] decharms RC, Christoff K, Glover GH, Pauly JM, Whitfield S, Gabrieli JDE. Learned regulation of spatially localized brain activation using real-time fMRI. Neuroimage 2004; 21: 436-443.

[8] Sitaram R, Caria A, Veit R, Gaber T, Kuebler A, Birbaumer N. Real-time fMRI based braincomputer interface enhanced by interactive virtual worlds, in: 45th Annual Meeting Society for Psychophysiological Research 2005; (Lisbon, Portugal). 
[9] Yoo SS, Jolesz FA. Functional MRI for neurofeedback: feasibility study on a hand motor task. Neuroreport 2002; 13: 1377-1381.

[10] Sitaram R, Caria A, Veit R, Gaber T, Rota G, Kuebler A, Birbaumer N. FMRI brain-computer interface: a tool for neuroscientific research and treatment. Comput Intell Neurosci. 2007: 25487. doi: $10.1155 / 2007 / 25487$.

[11] Sokunbi MO, Linden DEJ, Habes I, Johnston S, Ihssen N. Real-time fMRI brain-computer interface: development of a "motivational feedback" subsystem for the regulation of visual cue reactivity. Front. Behav. Neurosci. 2014; 8: 392. doi: 10.3389/fnbeh.2014.00392.

[12] DiStefano III JJ, Stubberud AR, Williams IJ. Schaum's outline of theory and problems of feedback and control systems. $2^{\text {nd }}$ ed. New York: McGraw-Hill 1967.

[13] Abdelzaher T, Diao Y, Hellerstein JL, Lu C, Zhu X. Introduction to Control Theory And Its Application to Computing Systems. In: Liu Z, Xia CH, Ed. Part II, Performance Modeling and Engineering. New York: Springer US. 2008: pp 185 -215.

[14] Astrom KJ, Murray R. Feedback Systems: An Introduction for Scientists and Engineers. New Jersey; Princeton University Press 2008.

[15] Lipsitz LA. Physiological Complexity, Aging, and the Path to Frailty. Sci. Aging Knowl. Environ. 2004 Vol. 2004; Issue 16: p. pe16.

[16] Kouijzer MEJ, de Moor JMH, Gerrits BJL, Buitelaar JK, van Schie HT. Long-term effects of neurofeedback treatment in autism. Research in Autism Spectrum Disorders 2009; 3(2): 496-501.

[17] Friel PN. EEG biofeedback in the treatment of attention deficit hyperactivity disorder. Altern Med Rev. 2007; 12(2): 146-51.

[18] Machado S, Almada LF, Annavarapu RN. Progress and Prospects in EEG-Based BrainComputer Interface: Clinical Applications in Neurorehabilitation. Journal of Rehabilitation Robotics 2013; 1: 28-41. 
[19] Hirshberg LM, Chiu S, Frazier JA. Emerging brain-based interventions for children and adolescents: overview and clinical perspective. Child Adolesc Psychiatr Clin N Am. 2005; 14(1): 1-19.

[20] Sterman MB, Egner T. Foundation and practice of neurofeedback for the treatment of epilepsy. Appl Psychophysiol Biofeedback. 2006; 31(1): 21-35.

[21] Hammond DC. Neurofeedback with anxiety and affective disorders. Child Adolesc Psychiatr Clin N Am. 2005; 14(1): 105-23.

[22] Gruzelier J, Egner T. Critical validation studies of neurofeedback. Child Adolesc Psychiatr Clin N Am. 2005; 14(1): 83-104.

[23] Sulzer J, Haller S, Scharnowski F, Weiskopf N, Birbaumer N, Blefari ML, Bruehl AB, Cohen LG, DeCharms RC, Gassert R, Goebel R, Herwig U, LaConte S, Linden D, Luft A, Seifritz E, Sitaram R. Real-time fMRI neurofeedback: progress and challenges. Neuroimage. 2013; 76: 386-99.

[24] Johnson KA, Hartwell K, LeMatty T, Borckardt J, Morgan PS, Govindarajan K, Brady K, George MS. Intermittent "real-time" fMRI feedback is superior to continuous presentation for a motor imagery task: a pilot study. J. Neuroimaging 2012; 22 (1): 58-66.

[25] deCharms RC, Maeda F, Glover GH, Ludlow D, Pauly JM, Soneji D, Gabrieli JD, Mackey SC. Control over brain activation and pain learned by using real-time functional MRI. Proc Natl Acad Sci U S A. 2005; 102(51): 18626-31.

[26] Ihssen N, Sokunbi MO, Lawrence AD, Lawrence NS, Linden DEJ. Neurofeedback of visual food cue reactivity: a potential avenue to alter incentive sensitization and craving. Brain Imaging and Behavior 2016; doi:10.1007/s11682-016-9558-x.

[27] Habes I, Rushton S, Johnston SJ, Sokunbi MO, Barawi K, Brosnan M, Daly T, Ihssen N, Linden DEJ. fMRI neurofeedback of higher visual areas and perceptual biases. Neuropsychologia 2016; 85: 208-215.

[28] Caria A, Veit R, Sitaram R, Lotze M, Weiskopf N, Grodd W, Birbaumer N. Regulation of anterior insular cortex activity using real-time fMRI. Neurolmage 2007; 35 (3): 1238-1246. 
[29] LaConte SM. Decoding fMRI brain states in real-time. Neuroimage. 2011; 56(2):440-54.

[30] Linden DE, Habes I, Johnston SJ, Linden S, Tatineni R, Subramanian L, Sorger B, Healy D, Goebel R. Real-time self-regulation of emotion networks in patients with depression. PLoS One 2012; 7(6):e38115. doi: 10.1371/journal.pone.0038115.

[31] Goebel R. Cortex-based real-time fMRI. Neuroimage 2001; 13: S129-S129.

[32] Lang PJ, Bradley MM, Cuthbert BN. International affective picture system (IAPS): affective ratings of pictures and instruction manual. University of Florida 2005: Gainesville (FL).

[33] Peirce JW. PsychoPy - Psychophysics software in Python. J Neurosci Methods 2007; 162: 8 -13.

[34] Mayberg HS, Lozano AM, Voon V, McNeely HE, Seminowicz D, Hamani C, Schwalb JM, Kennedy SH. Deep brain stimulation for treatment-resistant depression. Neuron 2005; 45: $651-660$.

[35] Gruzelier J, Egner T, Vernon D. Validating the efficacy of neurofeedback for optimizing performance. Progress in Brain Research 2006; 159: 421-431.

[36] Scharnowski F, Hutton C, Josephs O, Weiskopf N, Rees G. Improving visual perception through neurofeedback. Journal of Neuroscience 2012; 32: 17830-17841.

[37] Ruiz S, Lee S, Soekadar SR, Caria A, Veit R, Kircher T, Birbaumer N, Sitaram R. Acquired self control of insula cortex modulates emotion recognition and brain network connectivity in schizophrenia. Hum Brain Mapp. 2013; 34(1):200-12.

[38] Hartwell KJ, Prisciandaro JJ, Borckardt J, Li X, George MS, Brady KT. Real-time fMRI in the treatment of nicotine dependence: a conceptual review and pilot studies. Psychol Addict Behav. 2013; 27(2): 501-9.

[39] Beauregard $M$, Lévesque J. Functional magnetic resonance imaging investigation of the effects of neurofeedback training on the neural bases of selective attention and response inhibition in children with attention-deficit/hyperactivity disorder. Appl Psychophysiol Biofeedback. 2006; $31(1): 3-20$.

[40] Sitaram R, Veit R, Stevens B, Caria A, Gerloff C, Birbaumer N, Hummel F. Acquired control of 
ventral premotor cortex activity by feedback training: an exploratory real-time FMRI and TMS study. Neurorehabil Neural Repair. 2012; 26(3):256-65.

[41] Haller S, Birbaumer N, Veit R. Real-time fMRI feedback training may improve chronic tinnitus. Eur Radiol. 2010; 20(3):696-703.

[42] Frank S, Lee S, PreissI H, Schultes B, Birbaumer N, Veit R. The obese brain athlete: selfregulation of the anterior insula in adiposity. PLoS One. 2012; 7(8): e42570.

[43] deCharms RC. Reading and controlling human brain activation using real-time functional magnetic resonance imaging. Trends Cogn Sci. 2007; 11(11): 473-81.

[44] Weiskopf N, Sitaram R, Josephs O, Veit R, Scharnowski F, Goebel R, Birbaumer N, Deichmann R, Mathiak K. Real-time functional magnetic resonance imaging: methods and applications. Magn Reson Imaging. 2007; 25(6): 989-1003.

[45] Fovet T, Jardri R, Linden D. Current Issues in the Use of fMRI-Based Neurofeedback to Relieve Psychiatric Symptoms. Curr Pharm Des. 2015; 21(23):3384-94.

[46] Cohen Kadosh K, Luo Q, de Burca C, Sokunbi MO, Feng J, Linden DE, Lau JY. Using real-time $f M R I$ to influence effective connectivity in the developing emotion regulation network. Neuroimage. 2016; 125: 616-26.

[47] Fovet T, Orlov N, Dyck M, Allen P, Mathiak K, JARDRI R. Translating Neurocognitive Models of Auditory-Verbal Hallucinations into Therapy: Using real-time fMRI-neurofeedback to treat voices. Frontiers in Psychiatry 2016; doi: 10.3389/fpsyt.2016.00103.

[48] Gerin MI, Fichtenholtz H, Roy A, Walsh CJ, Krystal JH, Southwick S, Hampson M. Real-time fMRI neurofeedback with war veterans with chronic PTSD: a feasibility study. Frontiers in Psychiatry 2016; doi: 10.3389/fpsyt.2016.00111.

[49] Zotev V, Phillips R, Yuan H, Misaki M, Bodurka J. Self-regulation of human brain activity using simultaneous real-time fMRI and EEG neurofeedback. Neuroimage. 2014; 85 Pt 3: 985-95. 
Fig. 1: Schematic representations of a process and control system. (A) A process with input and output signals (B) When another block representing a controller is added to the process in (A), an open loop control system is created.

Fig. 2: Closed-loop feedback control system. The controller sends a control signal to the process which produces a controlled signal output. The feedback element extracts a feedback signal from the controlled signal output and sends it to a summer which detects an error signal from the difference between the reference input signal and the feedback signal. This error signal generates the control action of the system which serves as input to the controller, creating a feedback system.

Fig. 3: A closed-loop feedback control diagram for the human walking system. Here, the eyes represents the feedback element which sends feedback signal to the summing point where the error signal is detected from the difference between the desired and actual walk directions in a closed-loop fashion. When the eyes are closed the system becomes an open-loop system.

Fig. 4: fMRI neurofeedback architecture - localiser run. This is an open-loop set-up of the neurofeedback architecture which entails the signal acquisition and signal analysis subsystems using the GLM method. fMRI signals in response to a specific task are acquired from a participant by the signal acquisition subsystem (MRI scanner) and transferred in realtime to the signal analysis subsystem where signal processing, statistical analysis and the delineation of a ROI are done. The defined ROI is stored at the signal analysis subsystem for future neurofeedback runs. 
Fig. 5: fMRI neurofeedback architecture - neurofeedback run. This is a closed-loop set-up of the neurofeedback architecture where activated fMRI signals from a delineated ROI is acquired by the signal acquisition subsystem (MRI scanner) and transferred in real-time to the signal analysis subsystem. From here, the fMRI signals undergo a series of signal processing and statistical analysis before the feedback subsystem translates and presents the signals in visual form (e.g. thermometer bars) to the participant while still in the scanner. 